\title{
Colchicine inhibits pressure-induced tumor cell implantation within surgical wounds and enhances tumor-free survival in mice
}

\author{
David H. Craig, ${ }^{1}$ Cheri R. Owen, ${ }^{1}$ William C. Conway, ${ }^{1}$ Mary F. Walsh, ${ }^{1}$ \\ Christina Downey, ${ }^{1}$ and Marc D. Basson ${ }^{1,2,3}$ \\ 1Department of Surgery, 2Department of Anesthesiology, and 'Department of Anatomy and Cell Biology, \\ John D. Dingell VA Medical Center and Wayne State University, Detroit, Michigan, USA.
}

\begin{abstract}
Iatrogenic tumor cell implantation within surgical wounds can compromise curative cancer surgery. Adhesion of cancer cells, in particular colon cancer cells, is stimulated by exposure to increased extracellular pressure through a cytoskeleton-dependent signaling mechanism requiring FAK, Src, Akt, and paxillin. Mechanical stimuli during tumor resection may therefore negatively impact patient outcome. We hypothesized that perioperative administration of colchicine, which prevents microtubule polymerization, could disrupt pressurestimulated tumor cell adhesion to surgical wounds and enhance tumor-free survival. Ex vivo treatment of Co26 and Co51 colon cancer cells with colchicine inhibited pressure-stimulated cell adhesion to murine surgical wounds and blocked pressure-induced FAK and Akt phosphorylation. Surgical wound contamination with pressure-activated Co26 and Co51 cells significantly reduced tumor-free survival compared with contamination with tumor cells under ambient pressure. Mice treated with pressure-activated Co26 and Co51 cells from tumors preoperatively treated with colchicine in vivo displayed reduced surgical site implantation and significantly increased tumor-free survival compared with mice exposed to pressure-activated cells from tumors not pretreated with colchicine. Our data suggest that pressure activation of malignant cells promotes tumor development and impairs tumor-free survival and that perioperative colchicine administration or similar interventions may inhibit this effect.
\end{abstract}

\section{Introduction}

Viable tumor cells can frequently be recovered from the peritoneal cavity and from the portal and systemic venous circulation during colon cancer resections $(1,2)$. The presence of free malignant cells is a poor prognostic factor $(3,4)$, but how often these shed tumor cells cause perioperative metastasis is difficult to quantify (5-7). Wound recurrence occurs in $0.2 \%-1 \%$ of cases (8), and many patients exhibit other peritoneal spread at the time of this recurrence. Although peritoneal spreading and distant metastases identified after surgery may also reflect preoperative metastases that were too small to be detected, at least some recurrence is likely caused by the dissemination of tumor cells from the surgical procedure itself (9).

Metastatic progression depends on the initial adhesion of shed tumor cells to surrounding tissues. Exposure to forces including extracellular pressure, turbulence, and laminar and nonlaminar shear stimulates cancer cell adhesion to matrix proteins, endothelial cell monolayers, and surgical wounds in vivo by modulating integrin binding affinity through a mechanism requiring FAK, Src, Akt, and paxillin (10-15). Shed tumor cells may be subjected to such forces during vascular and lymphatic transit, in the tumor microenvironment, or iatrogenically through surgical manipulation, laparoscopic insufflation, and postoperative bowel edema (16-20). Cells from colon cancer lines, murine colonic adenocarcinomas, primary human colon cancers, head and neck squa-

Nonstandard abbreviations used: MTT, 3-(4, 5-dimethylthiazolyl-2)-2,5-diphenyltetrazolium bromide; PCNA, proliferative cell nuclear antigen.

Conflict of interest: The authors have declared that no conflict of interest exists. Citation for this article: J. Clin. Invest. 118:3170-3180 (2008). doi:10.1172/JCI34279. mous cell cancers, and breast adenocarcinomas all display similar pressure-mediated phenomena $(10-12,21,22)$.

The primary mechanosensor that activates this pathway is unclear. We postulated that the cell cytoskeleton might be a crucial component in the biochemical translation and relay of the forcedriven signals influencing cell adhesion. A preliminary in vitro study suggested that pharmacologic alterations of cytoskeletal dynamics ablates the activation of cancer cell adhesion by pressure (23). Colchicine disrupts microtubule dynamics by irreversibly binding to tubulin dimers and preventing microtubule polymerization (24). We therefore hypothesized that a single perioperative dose of colchicine before tumor resection might reduce tumor cell adhesiveness, wound implantation, and tumor recurrence.

We tested this hypothesis in a previously characterized murine transplantable colon cancer model using 2 different transplantable colon cancer lines, Co26 and Co51 $(11,25)$. In this model, tumors are resected from donor mice, and free tumor cells are exposed to either ambient or elevated pressure conditions and placed in surgical wounds in recipient mice for 30 minutes before nonadherent cells are washed away and the wounds closed. We have previously demonstrated that a 30-minute exposure to $15 \mathrm{mmHg}$ increased extracellular pressure enhances murine Co26 and Co51 adenocarcinoma cell implantation to surgical wounds by $30 \%-60 \%$ compared with tumor cells maintained under ambient conditions (25). In the current study, tumor cells isolated from donor mice were initially treated with colchicine ex vivo to assess the effects of colchicine on pressure-mediated tumor cell wound implantation, peritoneal metastasis, and relevant mechanical signaling. In parallel studies, the donor mice themselves were pretreated with colchicine in vivo before tumor resection to more 


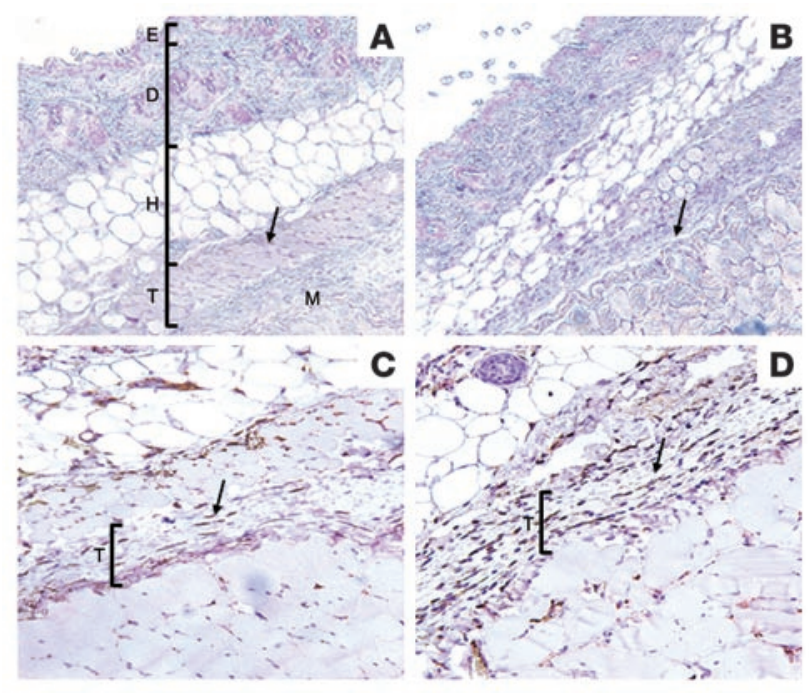

specifically examine whether a single systemic dose of this agent can block the pressure-activated adhesion pathway in cancers in situ. Although human cancer recurrences obviously occur in the same patients from whom the tumors have been resected, the division into donor and recipient mice allowed us to isolate the effects of colchicine pretreatment on the tumor itself from the effects of colchicine on the host tissues.

\section{Results}

Colchicine treatment inhibits pressure-stimulated tumor cell wound implantation. Consistent with our previous reports (25), immunohistological examination of murine surgical wounds contaminated with tumor cells exposed to either ambient pressure or increased pressure demonstrated increased implantation of pressure-activated cells (Figure 1). After exposure to ambient pressure or $15 \mathrm{mmHg}$ increased pressure, suspensions of HA-tagged murine colon tumor cells were equally dispersed over cutaneous abdominal wounds. After 30 minutes, wounds were thoroughly washed and closed with surgical staples. After 12 hours, fullthickness wounds were excised with 2-cm margins; fixed; paraffin embedded; sectioned to include epidermal, dermal, and muscular layers; and stained with anti-HA antibody to identify the tumor cells. Tumor cell implantation increased by $84 \% \pm 12 \%(n=6$, $P<0.01)$ in 6 random fields of 6 wounds exposed to pressuretreated tumor cells compared with those contaminated with cells maintained under ambient conditions.

We asked whether pressure-induced murine tumor cell wound implantation was sensitive to pretreatment with colchicine. Co26 and Co51 tumor cell suspensions were labeled with radioactive ${ }^{51} \mathrm{Cr}$, pretreated with $10 \mu \mathrm{M}$ colchicine or DMSO, and exposed to ambient or increased pressure. Equal aliquots of cell suspension were placed in murine groin incisions for 30 minutes before the wounds were vigorously washed and excised. Relative radioactivity of the excised wounds was used to quantify cell implantation (Figure 2). Preexposure to $15 \mathrm{mmHg}$ increased pressure enhanced DMSO-treated Co26 and Co51 cell adhesion to murine wounds by $58 \% \pm 24 \%(n=15, P<0.03)$ and $55 \% \pm 21 \%(n=12, P<0.04)$, respectively, compared with the same cells under ambient pressure. In contrast, pressure did not alter wound implantation of colchicine-treated cells in either cell line.

\section{Figure 1}

Tissue sections from murine cutaneous wound sites implanted with HA-tagged murine adenocarcinoma cells to observe tumor cell adherence. Arrows denote location between the hypodermis and the muscular layer, where adherent tumor cells were found when present. (A) Negative control wound site immunohistochemistry lacking primary $\mathrm{HA}$ antibody. $\mathrm{E}$, epidermis; $\mathrm{D}$, dermis; $\mathrm{H}$, hypodermis (mainly adipose tissue); T, tumor cell layer; M, muscular layer. (B) Tissue sections at the wound site lacking tumor cells stained for HA. (C) Wound site immunohistochemistry with HA-tagged tumor cells present between the hypodermis and muscular layer. (D) Wound site with HA-tagged tumor cells exposed to $15 \mathrm{mmHg}$ increased pressure for 30 minutes before implantation. Original magnification, $\times 100(\mathbf{A}$ and $\mathbf{B}) ; \times 200(\mathbf{C}$ and D).

To determine whether the inhibition of pressure-stimulated wound implantation by colchicine reflected microtubule disruption or some potential nonspecific effect unique to colchicine, we further assessed tumor cell wound implantation after pretreatment with 2 alternate microtubule-perturbing agents, vinblastine and rhizoxin, each targeting a different region of tubulin (26). Although these alternate microtubule inhibitors caused moderate differences in basal adhesion, pretreatment with vinblastine and rhizoxin abolished all pressure-mediated effects on Co26 and Co51 wound implantation, similar to the effects of colchicine pretreatment (Figure 2).

Colchicine inhibits pressure-induced peritoneal metastasis. Tumor cells shed during abdominal cancer surgery pose a risk not only for wound site implantation, but also for peritoneal metastasis. We therefore evaluated the effect of colchicine on pressure-induced tumor implantation in the peritoneal cavity (Figure 3). Murine CT26.WT cells, clones originally derived from the Co26 tumor line, were stably transfected with plasmid DNA encoding the luciferase enzyme (CT26.luc cells). CT26.luc cells were pretreated with colchicine or DMSO and exposed to either ambient pressure or increased pressure. The cells were then placed in the open peritoneal cavities of anesthetized mice. After 30 minutes, nonadherent cells were removed by thorough irrigation, and the abdomens were closed. At 10 days after surgery, mice were assessed for relative peritoneal spread and tumor burden through measurement of luciferase activity by bioluminescence. Mice exposed to pressure-activated CT26.luc cells displayed a tumor burden $49 \% \pm 19 \%$ larger $(n=8$, $P<0.05)$ than that of mice treated with CT26.luc cells maintained under ambient pressure. As we observed in our above-described wound implantation studies, colchicine pretreatment completely blocked pressure-stimulated peritoneal metastasis.

Colchicine inhibits pressure-stimulated phosphorylation of FAK and Akt, but not of Src and paxillin. We next evaluated the impact of colchicine treatment on pressure-stimulated phosphorylation of FAK, Src, Akt, and paxillin in Co26 tumor cells (Figure 4). Consistent with phosphorylation trends in human colon cancer lines, pressure stimulated a $28 \% \pm 7 \%$ increase in FAK Y397 phosphorylation and a $26 \% \pm 6 \%$ increase in FAK Y576 phosphorylation $(n=8, P<0.04$ for both; Figure 4, A and B). Pressure also induced a $29 \% \pm 8 \%(n=8$, $P<0.05)$ increase in Src Y416 phosphorylation and a $28 \% \pm 6 \%$ 

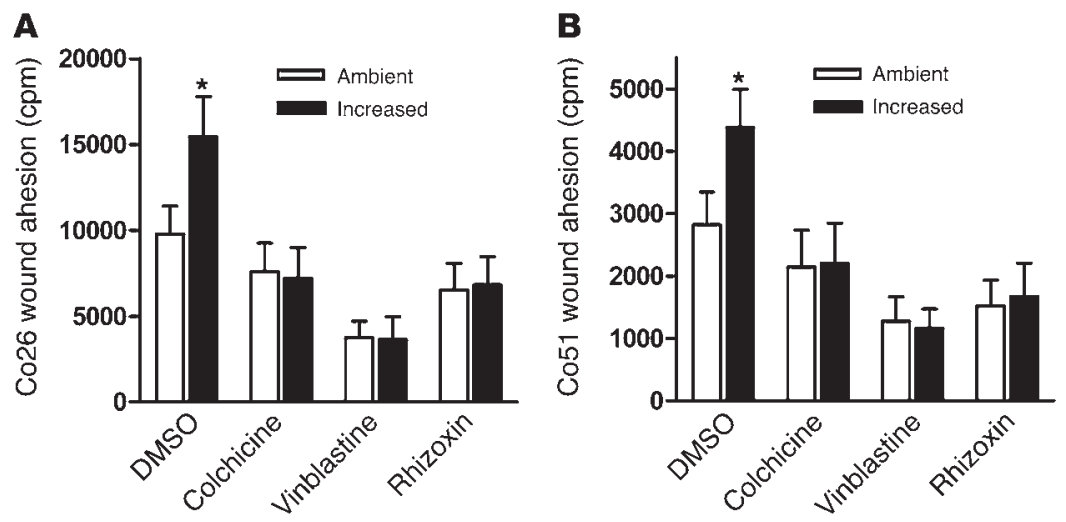

\section{Figure 2}

Effect of colchicine on pressure-mediated tumor cell wound implantation. ${ }^{51} \mathrm{Cr}$-labeled Co26 (A) and Co51 (B) tumor cells were pretreated with $10 \mu \mathrm{M}$ colchicine, $10 \mu \mathrm{M}$ vinblastine, $10 \mu \mathrm{M}$ rhizoxin, or DMSO and exposed to either ambient pressure or $15 \mathrm{mmHg}$ increased pressure and assessed for adhesion to murine surgical wounds. Tumor cell implantation was determined by the acquired radioactive $\mathrm{cpm}$ of surgical wounds after 30 minutes. Experiments were performed in a paired fashion, and data are graphically expressed as mean \pm SEM. ${ }^{*} P<0.05$ compared with respective ambient pressure control.

$(n=10, P<0.03)$ increase in Akt S473 phosphorylation (Figure 4, $\mathrm{C}$ and D). Paxillin Y31 phosphorylation was similarly increased by $30 \% \pm 7 \%$ under elevated pressure conditions $(n=12, P<0.03$; Figure $4 \mathrm{E})$. Phosphorylation trends in untreated cells did not differ from those in cells treated with a DMSO vehicle control.

Ex vivo Co26 treatment with $10 \mu \mathrm{M}$ colchicine completely blocked pressure-induced phosphorylation of FAK Y397 and FAK Y576 residues. Pressure-stimulated Akt S473 phosphorylation was similarly inhibited. In contrast to FAK and Akt phosphorylation, Src Y416 phosphorylation increased in response to pressure despite colchicine pretreatment $(33 \% \pm 9 \% ; n=8$, $P<0.05)$. Interestingly, treatment with colchicine increased basal paxillin Y31 phosphorylation by $24 \% \pm 6 \%(n=12, P<0.05)$ and failed to block a further $11 \% \pm 9 \%$ increase in phosphorylation in response to pressure.

Pressure promotes tumor cell adhesion through a mechanical signaling pathway in which FAK is both upstream and downstream of Akt. Because colchicine blocked pressure-induced FAK and Akt phosphorylation, we sought to further elucidate the role of FAK and Akt in this pathway to illuminate the mechanism of action of colchicine. We assessed pressure-stimulated CT26.WT cell adhesion to collagen I after pharmacologic inhibition of Akt and siRNA-mediated reduction of FAK. We further assessed whether expression of constitutively active myristoylated Akt (CT26.myr-Akt) could rescue cells from the inhibitory effects of colchicine (Figure 5A). Consistent with our results described above, a 30-minute exposure to elevated pressure increased CT26.WT cell adhesion by $32 \% \pm 9 \%(n=12, P<0.01)$ compared with cells under ambient pressure conditions. Pretreatment with colchicine blocked the effect. Functional inhibition of Akt using Akt Inhibitor VII (27) significantly reduced basal cell adhesion by $45 \% \pm 14 \%(n=12, P<0.02)$ and abolished any pressure-mediated effect. Likewise, basal levels of adhesion were reduced $39 \% \pm 7 \%$ $(n=12, P<0.01)$ by siRNA-mediated reduction of FAK, and no pressure effect was observed. Stably transfected CT26.myr-Akt cells adhered to collagen I $35 \% \pm 6 \%$ more than did CT26.WT controls. CT26.myr-Akt cell adhesion was further increased by $16 \% \pm 4 \%(n=12$, $P<0.05)$ under elevated pressure. Interestingly, colchicine failed to block pressure-stimulated adhesion $(19 \% \pm 7 \%$ increase; $n=12$, $P<0.05)$ in cells expressing myristoylated Akt, whereas CT26.myrAkt cell transfection with FAK-specific siRNA inhibited this effect.

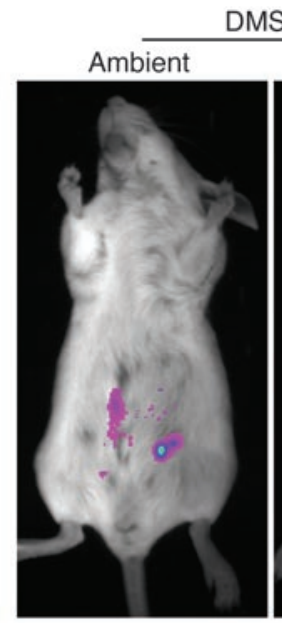

DMSO

Increased
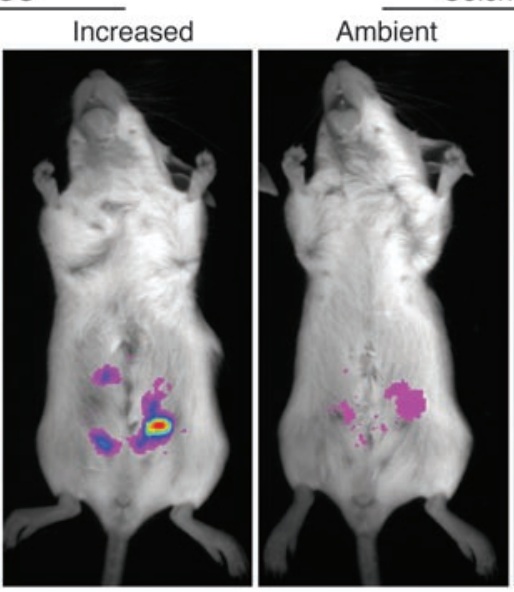
Increased
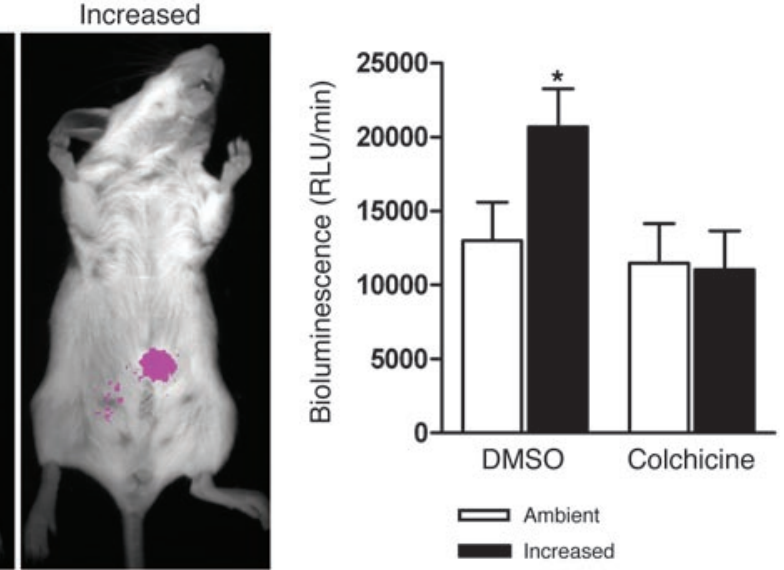

Figure 3

Effect of colchicine on pressure-induced i.p. metastasis. CT26.luc cells were pretreated with $10 \mu \mathrm{M}$ colchicine or DMSO and exposed to either ambient pressure or $15 \mathrm{mmHg}$ increased pressure. Tumor cell suspensions were dispersed throughout the peritoneal cavity, and nonadherent cells were washed away after 30 minutes. Relative i.p. metastasis was determined by CT26.luc bioluminescence 10 days after surgery. Bioluminescence images were pseudocolored (pink, least intense; red, most intense) and quantified for mean intensity over a fixed area. Calculated values were expressed as RLU per minute. Experiments were performed in a paired fashion, and data are graphically expressed as mean \pm SEM. ${ }^{*} P<0.05$ compared with respective ambient pressure control. 
A

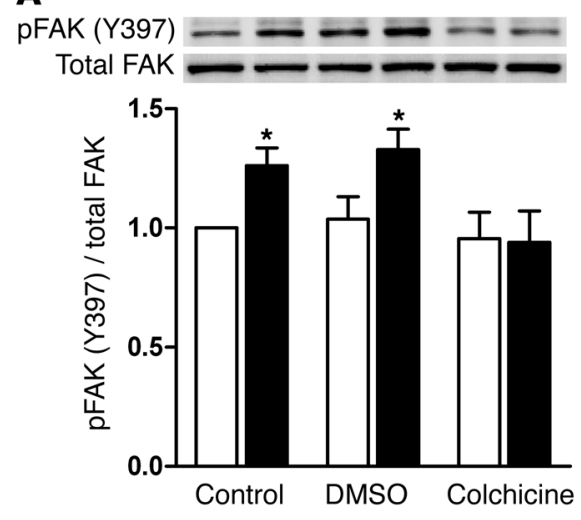

ש Ambient Increased

D
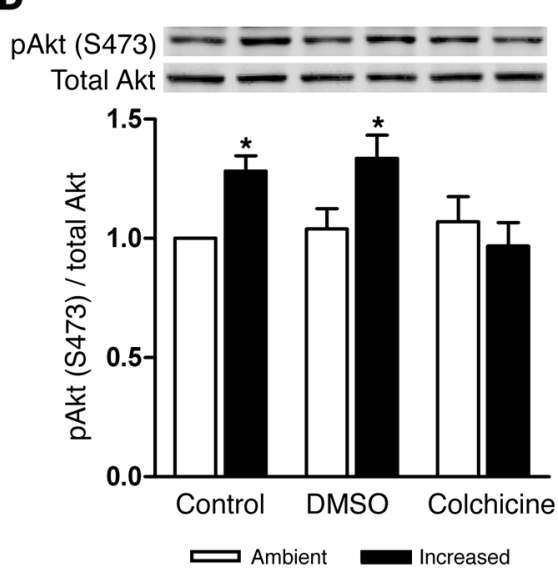

B
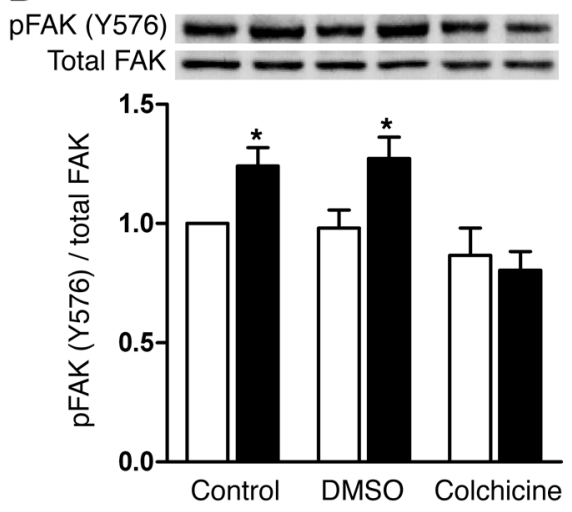

ב Ambient
C pSrc (Y416)

Total Src

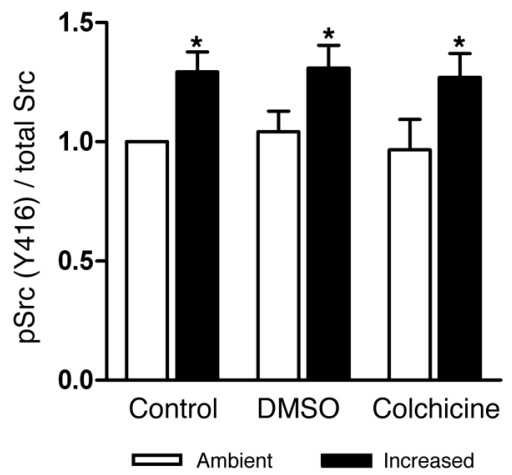

$\mathbf{E}$
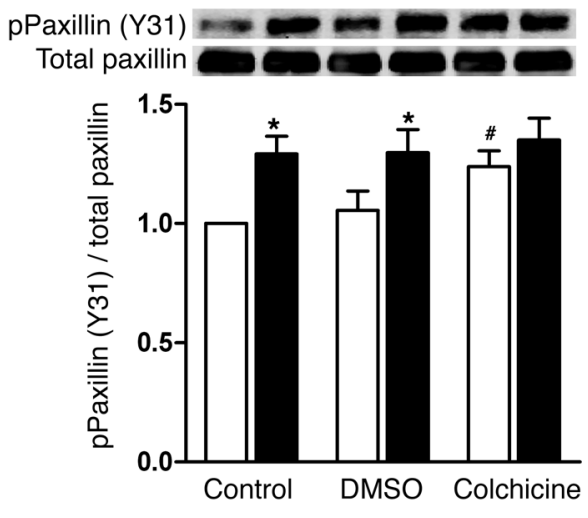

Ambient $\square$ Increased

Figure 4

Effect of ex vivo colchicine treatment on pressure-activated FAK, Src, Akt, and paxillin phosphorylation. Suspended Co26 tumor cells were treated ex vivo with either $10 \mu \mathrm{M}$ colchicine or DMSO vehicle control. Untreated Co26 cells (Control) were used as an additional control against DMSO. Cells were exposed to either ambient pressure or $15 \mathrm{mmHg}$ increased pressure, lysed, and assessed by Western blot for FAK, Src, Akt, and paxillin phosphorylation. Shown are the effects of colchicine on pressure-stimulated FAK Y397 (A), FAK Y576 (B), Src Y416 (C), Akt S473 (D), and paxillin Y31 (E) phosphorylation. Data from individual experiments were normalized to respective untreated controls exposed to ambient pressure and are graphically expressed as mean \pm SEM. ${ }^{*} P<0.05$ compared with respective ambient pressure control. ${ }^{\#} P<0.05$ compared with untreated ambient pressure control.

We assessed Akt S473 and FAK Y397 phosphorylation under these conditions in parallel (Figure 5, B and C). Consistent with the parental Co26 tumor line, pressure increased Akt S473 phosphorylation by $36 \% \pm 9 \%(n=9, P<0.03)$ and FAK Y397 phosphorylation by $25 \% \pm 6 \%(n=9, P<0.05)$ in CT26.WT cells. Colchicine inhibited these effects. As expected, pretreatment with $50 \mu \mathrm{M} \mathrm{Akt}$ Inhibitor VII reduced basal Akt S473 phosphorylation by $62 \% \pm 9 \%$ $(n=9, P<0.02)$ and similarly blocked Akt phosphorylation under pressure. Inhibition of Akt modestly affected basal levels of FAK phosphorylation, but completely blocked increased FAK Y397 phosphorylation under pressure. FAK reduction by siRNA also disrupted pressure-induced Akt phosphorylation. CT26.myr-Akt cells displayed a 2-fold increase in Akt S473 phosphorylation compared with CT26.WT cells. A significant increase in pressure-stimulated Akt phosphorylation was not discernible in these cells as a result of the extreme elevation in basal Akt phosphorylation. Of note, Akt phosphorylation in CT26.myr-Akt cells was modestly affected by colchicine and remained above levels found in pressure-treated CT26.WT cells. Furthermore, expression of myristoylated Akt rescued pressure-induced FAK Y397 phosphorylation $(24 \% \pm 8 \%$; $n=9, P<0.05)$ following treatment with colchicine.

Perioperative in vivo administration of colchicine in hibits pressure-stimulated tumor cell signaling and wound implantation with efficacy similar to that of to ex vivo treatments. To strengthen the clinical relevance of these findings, we administered colchicine to donor mice with tumors preoperatively rather than adding the drug to the isolated cells ex vivo to mimic a potential preoperative prophylactic dosing. Colchicine ( $2 \mathrm{mg} / \mathrm{kg}$ i.p.) was injected into tumor-bearing donor mice 12 hours before tumor resection. Co26 cells were then isolated from the tumor, exposed to either ambient pressure or increased pressure, and were reexamined to determine their capacity to adhere to surgical wounds in recipient mice as well as relevant mechanical signaling trends. Consistent with our observations described above, DMSO-treated Co26 cells displayed a $41 \% \pm 18 \%$ increase in wound implantation under elevated pressure conditions $(n=10, P<0.05$; Figure $6 \mathrm{~A})$. In vivo colchicine administration blocked this effect. Pressure increased FAK Y397, FAK Y576, and Akt 4473 phosphorylation by $30 \% \pm 9 \%(P<0.05)$, 


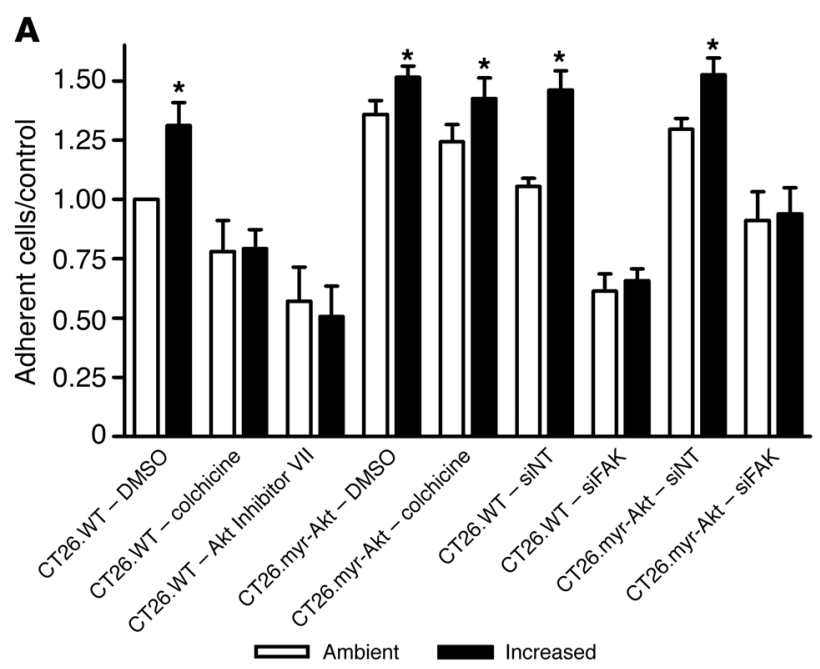

B
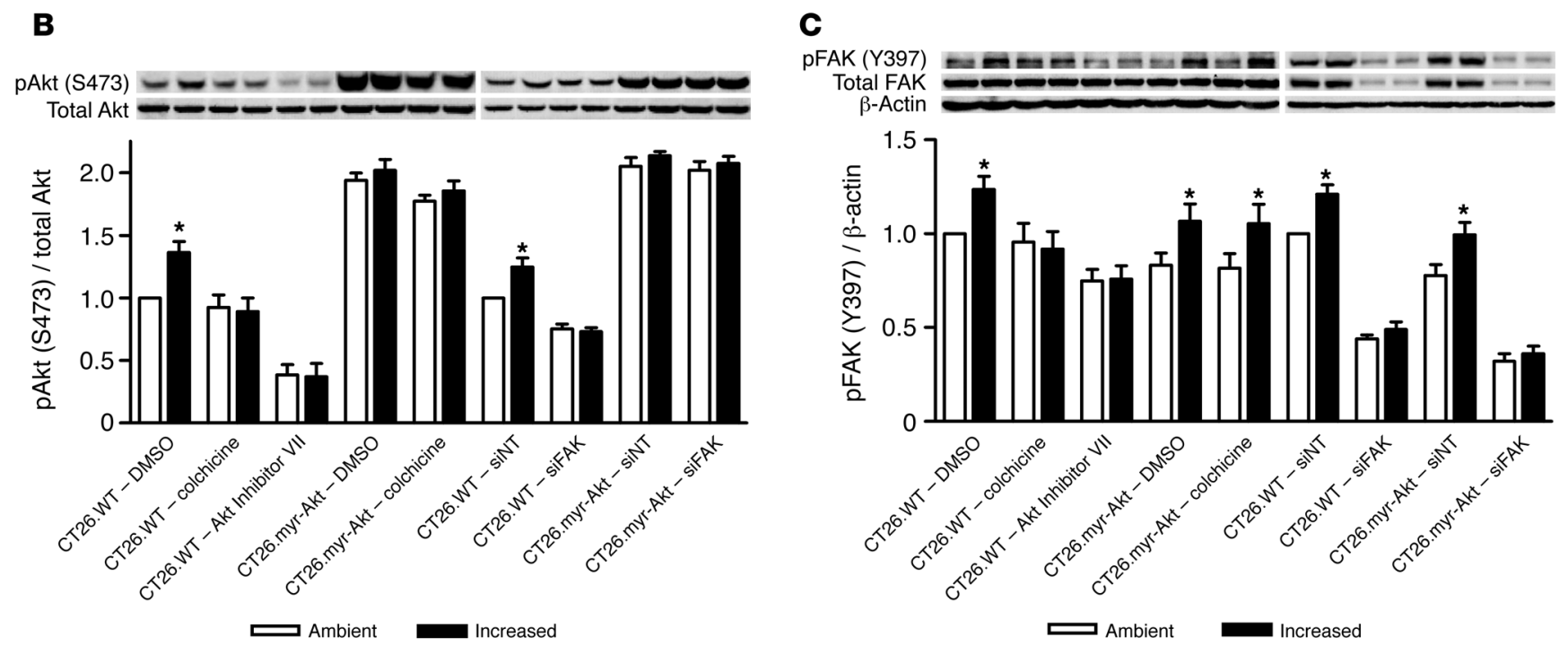

Figure 5

Effect of FAK and Akt manipulation on pressure-stimulated cell adhesion, signaling, and sensitivity to colchicine. siRNA-mediated reduction of FAK (siFAK), pharmacologic inhibition of Akt (Akt Inhibitor VII), and expression of CT26. myr-Akt was assessed in CT26.WT cells in conjunction with treatment with colchicine or DMSO and exposure to ambient pressure or $15 \mathrm{mmHg}$ increased pressure. (A) Effects of FAK and Akt manipulation on pressure-stimulated adhesion to collagen I. (B and C) Effects of FAK and Akt manipulation on pressure-induced Akt S473 (B) and FAK Y397 (C) phosphorylation. Data from individual experiments were normalized to respective ambient pressure DMSO-treated or nontargeting siRNA-treated (siNT) controls and graphically expressed as mean \pm SEM. ${ }^{*} P<0.05$ compared with respective ambient pressure control.

$34 \% \pm 7 \%(P<0.03)$, and $31 \% \pm 8 \%(P<0.05)$, respectively, in tumor cells isolated from DMSO-treated mice $(n=8$ for each; Figure 6 , $\mathrm{B}-\mathrm{D})$. In vivo colchicine administration inhibited pressureinduced FAK and Akt phosphorylation.

Perioperative colchicine administration antagonizes the negative impact of increased pressure on murine tumor-free survival. Although we had previously demonstrated that increased extracellular pressure can stimulate tumor cell adhesion to surgical wounds (25), it remained unknown whether the observed pressure-mediated increases in the number of cells adhering to these wounds would directly affect murine tumor development or tumor-free survival. As in the wound implantation studies, Co26 and Co51 tumor cells were isolated from tumor-bearing donor mice. After exposure to either ambient pressure or increased pressure, equal aliquots of tumor cell suspension were placed in surgical incisions in anesthetized mice. After 30 minutes, the wounds were thoroughly washed and closed. Mice were examined daily for palpable tumors, and growth was charted until a total tumor burden of $100 \mathrm{mg}$ was reached (Figure 7 ). Of the control mice, $52 \%$ of Co26 and $44 \%$ of Co51 developed tumors. On average, palpable tumors were observed by 20 days in both Co26 and Co51 control groups, and a 100-mg tumor burden was reached by days 34 and 33, respectively. The 100 -mg tumor burden was predetermined as a final end point in consultation with our veterinarians based upon animal welfare considerations. Tumors developed in $72 \%$ of the mice exposed to pressure-activated Co26 cells. Similarly, $68 \%$ of mice exposed to pressure-activated Co51 cells developed tumors. The average tumor-free survival in Co26 and Co51 pressure-activated groups were reduced to 17 and 18 days, respectively, and maximal tumor burden was reached by 27 and 26 days, respectively. The log-rank 
A
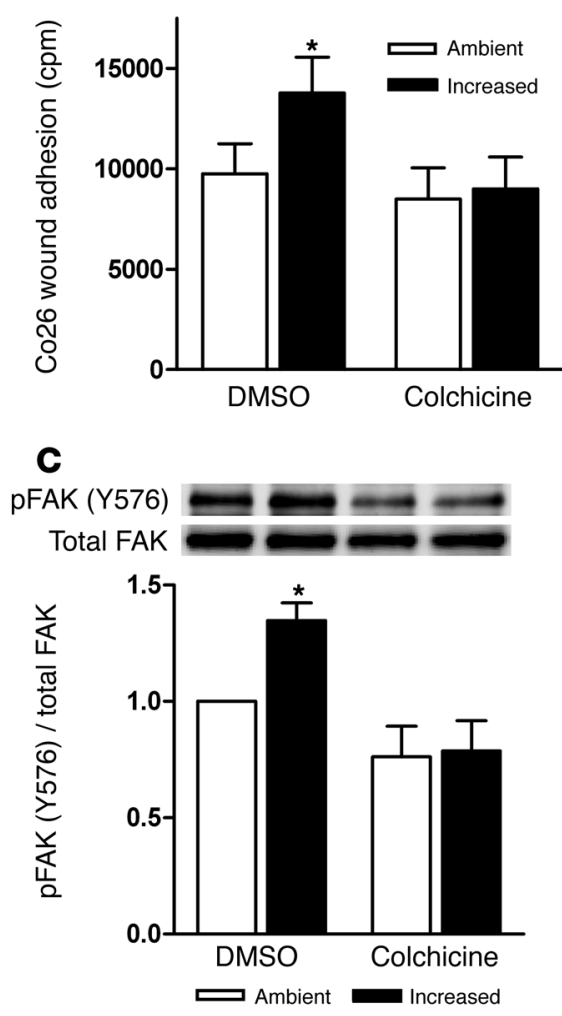

B
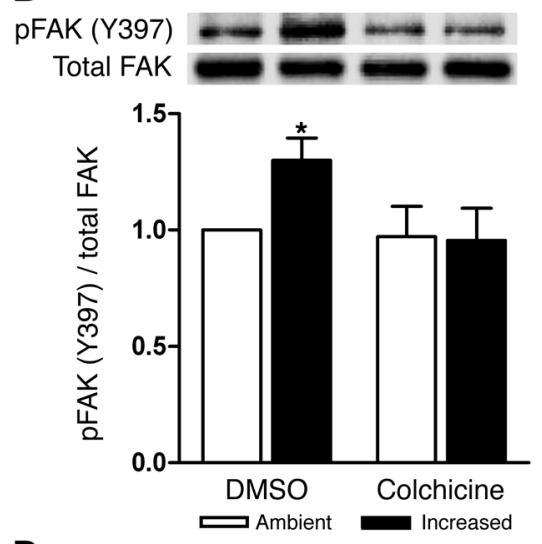

D
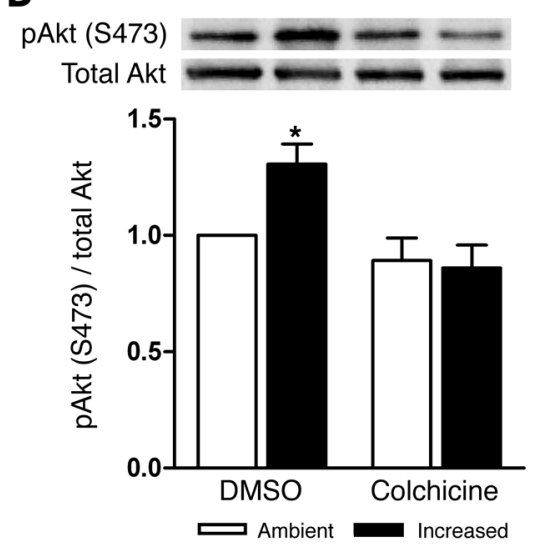

Figure 6

Effect of in vivo preoperative colchicine administration on pressure-activated tumor cell wound implantation and FAK and Akt phosphorylation. Tumor-bearing donor mice were administered either $2 \mathrm{mg} / \mathrm{kg}$ colchicine or equivalent DMSO vehicle control in PBS by i.p. injection before tumor resection. Digested Co26 cell suspensions were exposed to either ambient pressure or $15 \mathrm{mmHg}$ increased pressure and assessed for adhesion to surgical wounds as well as FAK and Akt phosphorylation by Western blot. (A) Effect of in vivo colchicine administration on wound implantation of ${ }^{51} \mathrm{Cr}$-labeled Co26 cells. Tumor cell adhesion was determined by the acquired radioactive $\mathrm{cpm}$ of surgical wounds. (B-D) Effect of colchicine on pressure-stimulated FAK Y397 (B), FAK Y576 (C), and Akt S473 (D) phosphorylation. Data from individual experiments were normalized to respective ambient pressure DMSO-treated controls. Results are graphically expressed as mean \pm SEM. ${ }^{*} P<0.05$ compared with respective ambient pressure control. statistic for the plot of time to palpable tumor suggests the difference in curves between control and pressure groups is statistically significant in each case $(n=25, P<0.05$; Figure $7, \mathrm{~A}$ and $\mathrm{C})$. The curves depicting average time to develop a 100-mg tumor burden correlated with the time to palpable tumor and were similarly deemed to be significantly different between ambient pressure and increased pressure groups for mice exposed to either Co26 or Co51 tumor cells ( $n=25, P<0.05$; Figure 7, B and D).

We examined the effect of colchicine on murine tumor-free survival in parallel. Colchicine was preoperatively administered to the donor mice by i.p. injection 12 hours before tumor resection. Tumors developed in 56\% of the colchicine-treated Co26 control mice and $44 \%$ of the colchicine-treated Co51 control mice. Palpable Co26 and Co51 tumors were observed on average at 23 and 25 days, respectively, and a 100 -mg tumor burden was reached by days 39 and 42, respectively. No significant difference was found between the survival curves of DMSO- and colchicine-treated control mice. Consistent with our wound implantation data, only $48 \%$ and $32 \%$ of the mice exposed to colchicine-treated pressureactivated Co 26 and Co51 cells developed tumors. The mean Co26 and Co51 tumor-free survival was 26 and 23 days, respectively, and maximum tumor burden was reached by 37 and 40 days, respectively. Differences between colchicine-treated cells exposed to ambient and increased pressure were not significant. However, the differences between the curves for DMSO- and colchicine-treated pressure-activated cells for both time to palpable tumor and time to 100 -mg tumor burden were found to be statistically significant by log-rank analysis ( $n=25, P<0.04$ for each).
Colchicine-mediated effects on tumor development and survival are indicative of reduced tumor cell implantation, not altered proliferation or cytotoxicity. Finally, we sought to address whether the altered rates of tumor development observed between experimental groups reflect only differences in cell adhesiveness during the surgical procedure or might also be influenced by colchicine's antimitotic effects. As in our survival studies, tumor-bearing donor mice were preoperatively treated with colchicine 12 hours before tumor resection. We first assessed in vivo BrdU incorporation in the Co26 tumors. For these studies, rather than resecting the tumors 12 hours after colchicine treatment, we then injected mice with $2 \mathrm{mg} \mathrm{BrdU}$ i.p. and assessed them for BrdU uptake after an additional 12 hours by flow cytometric analysis of $\mathrm{Co} 26$ cell suspensions. BrdU incorporation was similarly assessed after 96 hours. Short-term BrdU incorporation was reduced by $21 \% \pm 6 \%$ in Co26 tumors treated with colchicine versus those treated with DMSO $(n=3, P<0.05$; Figure $8 \mathrm{~A})$. However, by 96 hours, BrdU incorporation was indistinguishable between DMSO- and colchicine-treated cells. We confirmed that colchicine had no long-term effects on tumor growth by preparing histological sections of tumors 20 days after initiation and staining for proliferative cell nuclear antigen (PCNA). We calculated the proliferative index as the percentage of PCNA-positive cells. No significant differences were noted between cells treated with DMSO or with colchicine under ambient pressure or increased pressure $(n=3$; Figure $8 \mathrm{~B})$. To reconcile the differences between our short- and long-term observations, we further assessed Co26 cell proliferation from 24 to 96 hours after colchicine administration. After tumor cell isolation, cell suspensions were exposed to either ambient or increased pressure 
A
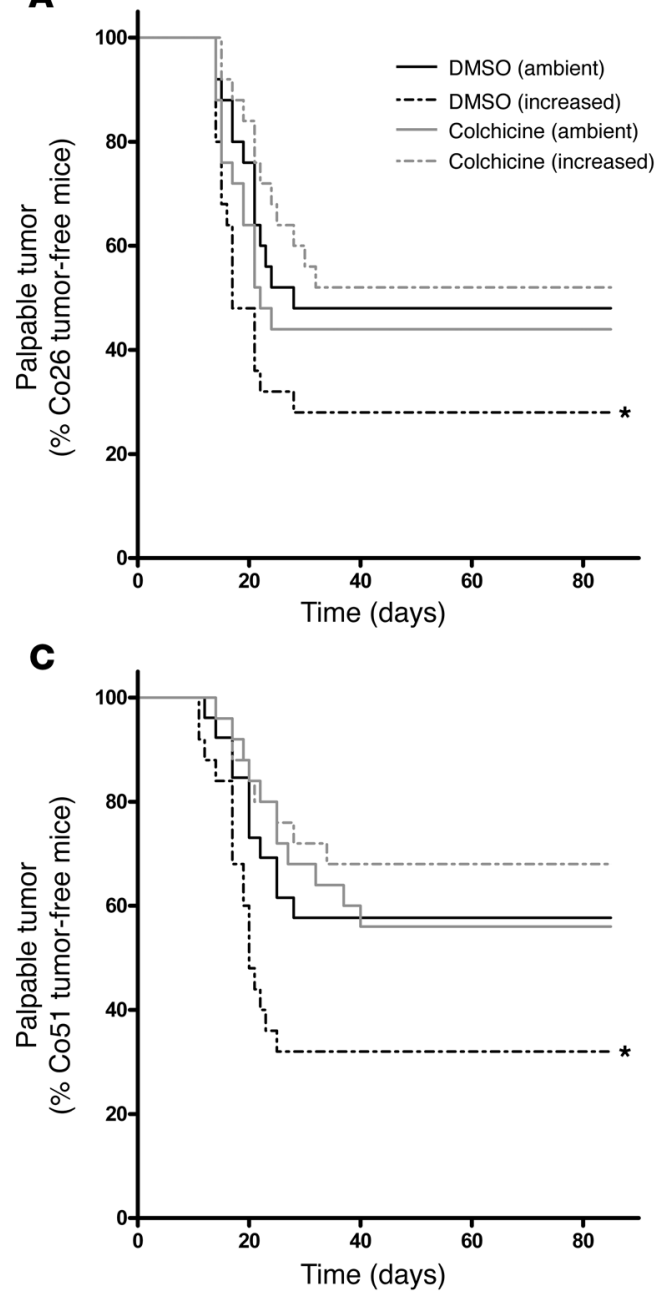

B

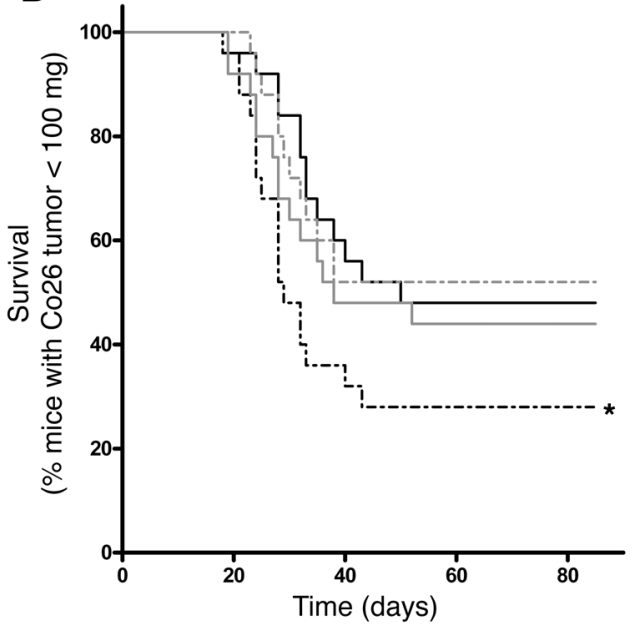

D

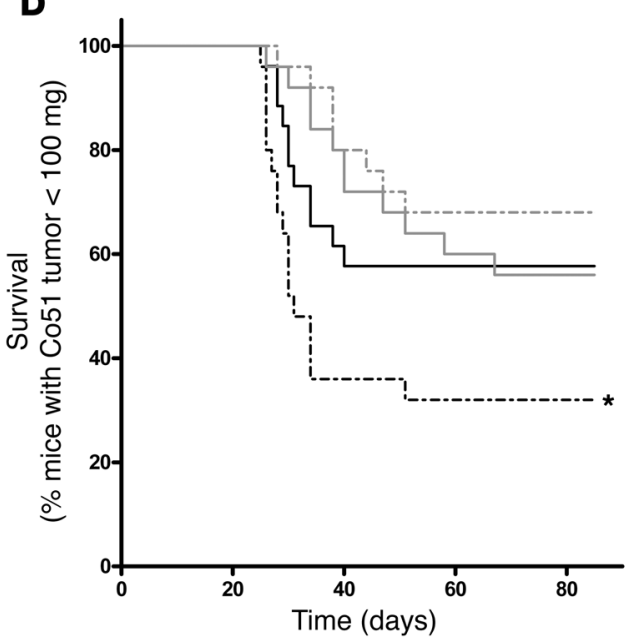

Figure 7

Effect of preoperative colchicine administration on murine tumor development and survival following exposure to increased pressure. Murine tumor development and survival were assessed after surgical wound contamination with $\mathrm{Co} 26$ and Co51 tumor cell suspensions preoperatively treated with either colchicine or DMSO and exposed to ambient pressure or $15 \mathrm{mmHg}$ increased pressure. Kaplan-Meier graphs depict ( $\mathbf{A}$ and $\mathbf{C})$ the incidence of palpable tumor development over time and ( $B$ and $\mathbf{D}$ ) the incidence of tumors reaching $100 \mathrm{mg}$ over time in $\mathrm{Co26}$ (A and $\mathbf{B}$ ) and Co51 (C and D) mice. The survival data were analyzed by log-rank test. ${ }^{*} P<0.05$ compared with DMSO-treated ambient pressure control. conditions for 30 minutes. Co26 cells were then lightly seeded and assessed for rates of proliferation by the colorimetric reduction of 3-(4, 5-dimethylthiazolyl-2)-2,5-diphenyltetrazolium bromide (MTT) over 24-hour increments ( $n=6$; Figure $8 \mathrm{C}$ ). The tumor cells proliferated more slowly for 48 hours after colchicine treatment. However, by 72 hours, proliferation was nearly identical between DMSO- and colchicine-treated cells. The ambient pressure and increased pressure groups did not differ significantly.

We further assessed the effects of pressure and colchicine on Co26 cell invasion to rule out potential differences in tumor invasiveness as a confounding variable in our survival studies. We assessed the invasion of isolated Co26 cells 24 hours after seeding on collagen I-coated membranes in simplified Boyden chambers. The Co26 cells were modestly invasive: $8 \% \pm 1 \%(n=3)$ of DMSOtreated control cells migrated through the matrix. Moreover, pressure did not affect invasion. At 24 hours after pretreatment with colchicine, cell invasion was reduced by approximately $2 \%$. No differences were observed between DMSO- and colchicine-treated Co26 cells after 96 hours (data not shown).

\section{Discussion}

The stimulation of cancer cell adhesion by extracellular pressure is both relevant to our understanding of tumor metastasis and important as a paradigm for mechanotransduction and inside-out signaling. Our present results demonstrate that pressure activation of malignant cells has a biological effect on tumor development and tumor-free survival in an animal model. In addition, our findings suggest that pharmacologic intervention may prevent this effect and reduce tumor recurrence. Finally, our results lend further support to the notion of the microtubule cytoskeleton as a crucial upstream mechanosensor in the activation of this signaling pathway.

The ability of relatively modest physical forces to profoundly alter cell biology has been well described in a variety of cell types and in response to different forces (28-31). How these forces initiate such responses without a classical ligand-receptor interaction is less clear. In various settings, stretch-activated ion channels, mechanosensitive membrane-associated enzymes, distortion of cytoskeletal filaments, and integrin-ECM interactions may all relay mechanical signals (32-36). Ingber's cellular tensegrity model, in which physical distortion of the cytoskeleton transfers mechanical loads through actin- and microtubule-associated molecules to initiate intracellular signaling, is consistent with the ability of colchicine to disrupt pressure-induced signals in our murine tumor model $(37,38)$.

Indeed, colchicine may exert 2 different effects on wound recurrence. Pretreatment of tumor-bearing mice with colchicine transiently inhibited tumor cell proliferation and modestly inhibited invasiveness independent of pressure. The antimitogenic effect was 
A

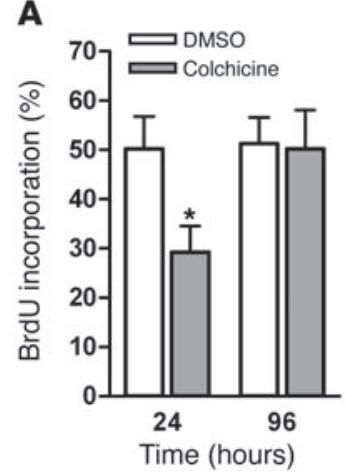

B

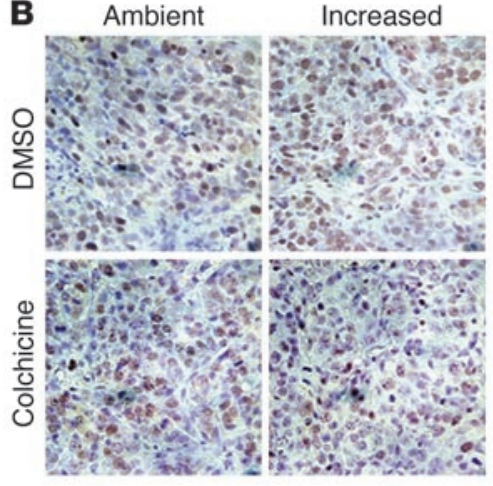

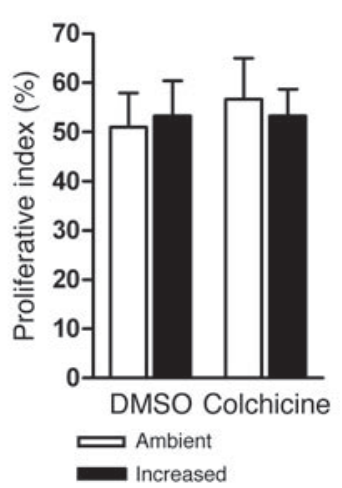

C

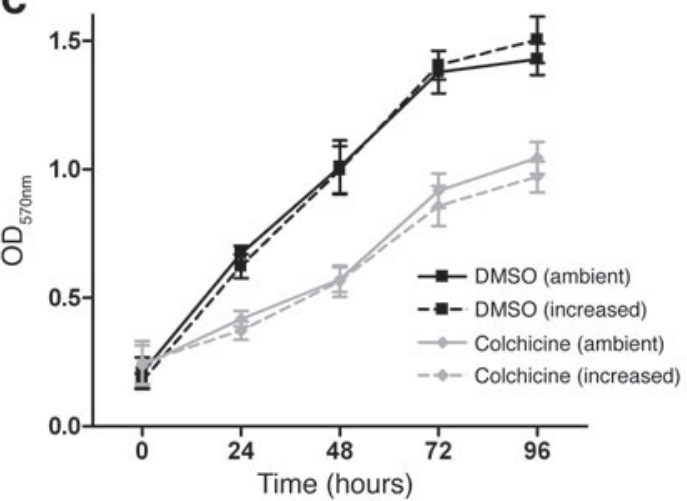

\section{Figure 8}

Effect of colchicine on tumor cell proliferation. Co26 tumor cell proliferation was assessed by BrdU incorporation, PCNA immunohistochemistry, and MTT reduction after i.p. colchicine treatment. (A) In vivo BrdU incorporation in Co26 tumors 24 and 96 hours after treatment with colchicine. (B) Immunohistology of PCNApositive tumor cells 20 days after implantation following treatment with colchicine or DMSO and exposure to either ambient pressure or $15 \mathrm{mmHg}$ increased pressure. Original magnification, $\times 40$. (C) At 12 hours after in vivo tumor treatment with colchicine or DMSO, isolated Co26 cells were exposed to either ambient pressure or $15 \mathrm{mmHg}$ increased pressure. In vitro cell proliferation of the respective populations was assessed by colorimetric analysis of MTT reduction every 24 hours. Data are graphically expressed as mean \pm SEM. ${ }^{*} P<0.05$ compared with respective DMSO-treated control. no longer detectable after 72 hours, and the anti-invasive effect is likely equally transient. This may explain the apparent tendency toward a 2- to 3-day lag in tumor development in mice exposed to colchicine-treated tumor cells in the absence of pressure compared with mice exposed to vehicle-treated tumor cells. Tumor proliferation was not altered by 30 minutes of pressure, consistent with our previous observation that 4.5 hours of pressure are required to stimulate proliferation in vitro (39). However, although pressure did not affect proliferation or invasion, colchicine nevertheless blocked the pressure effect on tumor development, consistent with our observations that colchicine blocked pressure-stimulated cell adhesion and associated pressure-activated signaling. This effect appeared independent of any effect of colchicine on proliferation or invasion and, in contrast to the antimitogenic effect, was reflected in an absolute and stable difference in tumor-free survival in this model, rather than the transient delay in tumor development that may be attributed to the antiproliferative and/or anti-invasive effect.

Consistent with previous observations in human colon cancer cell lines $(13,23)$, reducing FAK or inhibiting Akt blocked pressure-stimulated CT26.WT cell adhesion. The inhibition of pressure-induced FAK and Akt activation by preoperative colchicine suggests that a dynamic cytoskeleton is required for tumor cells to respond to extracellular pressure changes. The observation that siRNA-mediated FAK reduction inhibited pressure-stimulated Akt S473 phosphorylation, whereas Akt inhibition blocked pressurestimulated FAK Y397 phosphorylation, suggests that the relationship between FAK and Akt in this pathway is bidirectional. Previous studies demonstrated that Akt activation was required for FAK and Akt localization to the membrane as well as FAK- $\beta_{1}$-integrin interaction under elevated pressure conditions (13, 40). Myristoylated Akt expression rescued colchicine-mediated inhibition of FAK, yet FAK reduction blocked pressure-stimulated adhe- sion in CT26.myr-Akt cells. Taken together, these observations suggest that the cell cytoskeleton may relay pressure-induced signals through FAK upstream of Akt, and that FAK-dependent Akt activation is subsequently required for the maintenance of membrane-localized FAK activity critical for integrin activation and cell adhesion (Figure 9).

Although pressure-activated paxillin phosphorylation appears impervious to cytoskeletal perturbation, treating aortic endothelial cells with colchicine or paclitaxel increases paxillin tyrosine phosphorylation, which correlates with paxillin translocation away from focal adhesions into the cytoplasm (41). Thus, without more detailed investigation of the subcellular localization of paxillin in this setting, it is difficult to assess whether the observed elevation in paxillin phosphorylation following treatment with colchicine is functionally relevant.

In contrast to FAK and Akt, pressure-mediated Src activation has consistently proven cytoskeleton independent $(23,42)$. In the current study, colchicine similarly failed to block pressure-stimulated Src Y416 phosphorylation. Whether mechanical activation of Src is mediated by stretch-activated ion channels or some alternate pressure-sensitive molecule requires further study. We therefore cannot rule out a potential alternate or upstream mechanosensor separate from the cytoskeleton. Blockade of stretch-activated ion channels in rat chondrosarcoma cells can induce cytoskeletal reorganization without other stimuli (43). However, disruption of actin microfilaments or microtubules in renal epithelial cells eliminates shear stress-induced increases in intracellular calcium by inhibiting mechanosensitive ion channels (44). Regardless of the absolute primacy of the cytoskeleton or some other molecule in the sensation and relay of pressure-derived signals, microtubule disruption by colchicine appears sufficient to inhibit pressuremediated functional effects on cell adhesion. 


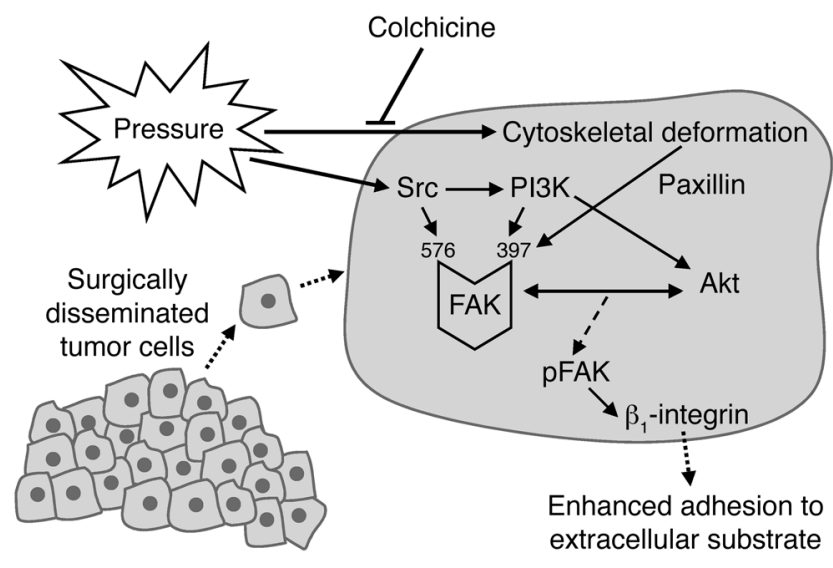

Figure 9

Working model of the intracellular signaling mechanism by which pressure stimulates tumor cell adhesion. Increased extracellular pressure appears to activate FAK through a complex dual pathway requiring an intact cytoskeleton and paxillin as well as cytoskeleton-independent activation of Src and PI3K. FAK is activated through phosphorylation at its Y397 and Y576 residues and in turn supports pressure-induced Akt S473 phosphorylation. Phosphorylated FAK localizes to $\beta_{1}$-integrin heterodimers in an Akt-dependent manner, resulting in integrin activation and increased substrate binding affinity.

The differences between this murine model and human colon cancer resection are multiple and manifest. To increase the power of our study with a realistic sample size, we specifically titered the number of tumor cells seeded per mouse to dramatically increase tumor recurrence over that observed after human colectomy for cancer. The actual baseline incidence of clinically significant tumor recurrence in surgical wounds is much lower, and it is impossible to reliably estimate the fraction of postoperative distant metastasis that reflects perioperative tumor dissemination. However, any reduction in the incidence of wound recurrence or distant dissemination would clearly be desirable. Prophylactic antibiotics are used to inhibit surgical wound infections after elective colectomy. Wound infections may occur an order of magnitude more frequently than isolated wound recurrences (45), but also have far less impact on long-term survival. Whether colchicine pretreatment or some other intervention directed at the pressure-stimulated signaling pathway regulating cancer cell adhesion could also reduce perioperative tumor adhesion and metastasis via the lymphatics and portal venous system awaits further study.

The parenteral colchicine dose used herein was consistent with colchicine dosing in other murine studies (46-48), but higher than that used to treat gout in humans. However, a single dose is likely to be better tolerated than repeated dosing over many days. Further studies would be required to determine how colchicine pharmacokinetics in humans compare with those in mice, the effect of treating recipient mice with colchicine, whether a lower colchicine dose similarly reduces tumor cell adhesion, and whether humans can tolerate a single preoperative dose of the magnitude used in the present study. Although chronic colchicine therapy can inhibit the healing of fractures, corneal epithelium, gastric mucosa, and contracting skin wounds (49-52), such effects from a single preoperative dose of colchicine are less likely, because most postsurgical wound healing would occur after the single dose of colchicine had been metabolized. Alternatively, other agents might target this adhesion-stimulating pathway.

Despite the relative rarity of wound recurrence, many other measures are proposed in human surgery to avoid wound recurrence of resected tumors. These include refined technique, mechanical irrigation, port site and intra-abdominal chemotherapy, and even the secondary excision of laparoscopic trocar wounds themselves (1,53-55). Unfortunately, little data support the efficacy of many such interventions. Dilute calcium chloride can also reduce tumor adhesion to murine surgical wounds and improve tumor-free survival (11). Divalent calcium ions can compete with magnesium in wound fluid for binding to an extracellular integrin divalent cation binding site that regulates integrin binding affinity, but calcium does not affect the intracellular force-activated pathway by which cancer cells regulate their own adhesion. A localized calcium wash would also not be expected to have any impact on vascular or lymphatic tumor dissemination. Whether colchicine and calcium could therefore synergistically reduce wound recurrence remains to be determined.

In summary, our present results suggest the possibility that cytoskeletal perturbation, whether by colchicine or by some other agent, can alter the responsiveness of malignant cells to external forces, reducing activation of FAK and Akt, inhibiting the subsequent adhesion of shed tumor cells, and enhancing tumor-free survival. Because epithelial tumor cells of other types, including breast (22) and head and neck (21), also increase adhesion in response to elevated extracellular pressure, our results may also have implications for malignancies other than colon cancer.

\section{Methods}

Mice. BALB/c mice were used for tumor propagation, implantation, and survival studies. Animal procedures were conducted with approval of the Wayne State University Animal Investigation Committee and the John D. Dingell VA Medical Center Research and Development Committee.

Tumor propagation and isolation of murine colon cancer cells. Co26 and Co51, transplantable murine colon adenocarcinomas (56), were passed in BALB/c mice by sterile subcutaneous injection. Mice were euthanized when tumors reached $1,000 \mathrm{mg}$. Tumors were excised sterilely from the abdominal wall, washed in PBS, finely minced in DMEM, and digested in DMEM containing $0.15 \%$ type IV collagenase at $37^{\circ} \mathrm{C}$ for 1 hour, or until a single cell suspension was achieved. The cells were again washed and resuspended in DMEM containing 5\% FBS as previously described (25). After isolation, Co26 and Co51 viability was assessed by trypan blue exclusion, and cells were directly used for experimentation.

CT26.WT cells, clones of the parental Co26 line, were purchased from ATCC, maintained at $37^{\circ} \mathrm{C}, 5 \% \mathrm{CO}_{2}$, and grown in RPMI- 1640 with $2 \mathrm{mM}$ L-glutamine, $10 \mathrm{mM}$ HEPES, $1 \mathrm{mM}$ sodium pyruvate, $4.5 \mathrm{~g} / \mathrm{l}$ glucose, $1.5 \mathrm{~g} / 1$ $\mathrm{NaHCO}_{3}$, and $10 \%$ FBS. CT26.luc and CT26.myr-Akt stable transfectants were cultured with $600 \mu \mathrm{g} / \mathrm{ml}$ geneticin (Invitrogen).

Transfection. Cells were transfected with $50 \mathrm{nM}$ double-stranded siRNA directed toward the murine mRNA target 5'-GGAAAUAUGAGUUGAGAAU-3' (Dharmacon) for FAK reduction. Dharmacon siCONTROL Non-Targeting siRNA no. 1 served as a control. siRNAs were introduced with Oligofectamine according to the manufacturer's protocol (Invitrogen). Cell transfectants were used for adhesion experiments after 48 hours.

Stable expression of luciferase by CT26.WT cells was achieved by cotransfection with the pGL3 luciferase reporter plasmid (Promega) and pcDNA3.1 (Invitrogen) for antibiotic resistance. CT26.WT cell transfection with pcDNA3.1 myr-Akt1 (Addgene) was used for stable expression of myristoylated Akt. Plasmids were introduced using Lipofectamine 2000 
(Invitrogen) per the manufacturer's protocol. Stable CT26.luc and CT26. myr-Akt clones were selected using geneticin.

Pressure regulation. Pressure was controlled using an airtight Lucite box with an inlet valve for gas application and an outlet valve connected to a manometer (10). The box was prewarmed to $37^{\circ} \mathrm{C}$ to prevent internal temperature and pressure fluctuations. Temperature was maintained within $2{ }^{\circ} \mathrm{C}$, and pressure maintained within $1.5 \mathrm{mmHg}$, of desired levels.

In vitro cell adhesion assay. Suspended cells were allowed to adhere to collagen I-coated 6-well plates $\left(10^{5}\right.$ cells/well) for 30 minutes at $37^{\circ} \mathrm{C}$ under ambient or $15 \mathrm{mmHg}$ increased pressure conditions. After 30 minutes, nonadherent cells were gently washed away with warm PBS, and adherent cells were formalin fixed, hematoxylin stained, and counted in at least 20 random high-power fields per well using an inverted microscope (10). Cell suspensions were pretreated for 30 minutes with $10 \mu \mathrm{M}$ colchicine (Sigma-Aldrich), $50 \mu \mathrm{M}$ Akt Inhibitor VII (Calbiochem), or DMSO vehicle control, and treatment was maintained during adhesion studies.

Wound implantation studies. Bilateral 2-cm groin incisions were made in mice anesthetized i.p. with $60 \mathrm{mg} / \mathrm{kg}$ pentobarbital. A 100- $\mu$ l suspension of $10^{5}{ }^{51} \mathrm{Cr}$-labeled tumor cells maintained under either ambient pressure or increased pressure was applied to each wound. The fluid was aspirated after 30 minutes, and the wounds were gently washed with PBS to remove nonadherent cells. In nonsurvival studies, the mice were euthanized after wound irrigation, and the wounds were excised immediately to quantify tumor adhesion by radioactivity in the excised tissue using an automated scintillation counter. In parallel survival studies using unlabeled cells, the wounds were closed after irrigation, and the animals were followed for 90 days for tumor development. The mice were monitored daily and sacrificed when tumors reached $100 \mathrm{mg}$. We measured the time to first palpable tumor and the time to achieve a $100-\mathrm{mg}$ tumor. Tumors were measured 5 times per week, and mass in milligrams was estimated from 2-dimensional measurements calculated as follows: $\left(a \times b^{2}\right) / 2$, where $a$ and $b$ represent tumor length and width, respectively, in millimeters (57). Tumor cells were treated with $10 \mu \mathrm{M}$ colchicine, $10 \mu \mathrm{M}$ vinblastine, or $10 \mu \mathrm{M}$ rhizoxin (Sigma-Aldrich) ex vivo 30 minutes before and for $30 \mathrm{~min}$ utes during implantation experiments or in vivo by $2 \mathrm{mg} / \mathrm{kg}$ i.p. colchicine injection of donor mice 12 hours before tumor resection. DMSO was used as a vehicle control.

Bioluminescent imaging of peritoneal metastases. One-inch abdominal incisions were made in mice anesthetized i.p. with $60 \mathrm{mg} / \mathrm{kg}$ pentobarbital. A $100-\mu \mathrm{l}$ suspension of $10^{6} \mathrm{CT} 26.1 \mathrm{luc}$ tumor cells treated with $10 \mu \mathrm{M}$ colchicine or DMSO and maintained under either ambient pressure or increased pressure was dispersed in the peritoneal cavity of each mouse. After 30 minutes, the abdominal cavities were thoroughly irrigated with PBS, and the excess fluid was aspirated to remove nonadherent cells. The abdominal wounds were then closed with sutures. After 10 days, mice were reanesthetized i.p. with $60 \mathrm{mg} / \mathrm{kg}$ pentobarbital and administered $150 \mathrm{mg} / \mathrm{kg}$ D-luciferin i.p. (BD Biosciences). After 10 minutes, mice were assessed for relative CT26.luc tumor bioluminescence using a Kodak IS4000MM small animal imaging device (Kodak) over 10 minutes. Exposure conditions (time, aperture, stage position, binning, and time after injection) were constant for all measurements. Bioluminescence intensity images were pseudocolored (pink, least intense; red, most intense) and quantified using MetaMorph software (version 7.0; Molecular Devices). A fixed region of interest was defined for all measurements for quantification of average image intensity. Calculated values were expressed as RLU per minute.

Western blotting. Suspended (nonadherent) cells were subjected to ambient pressure or increased pressure for 30 minutes in bacteriologic plastic dishes pretreated with $1 \%$ heat-inactivated bovine serum albumin to block cell adhesion. Cells were collected and lysed as previously described
(42). Cell lysate protein concentrations were determined by BCA protein assay (Pierce). Equal protein aliquots were resolved by SDS-PAGE and transferred to Hybond ECL nitrocellulose membrane (GE Healthcare). Mouse monoclonal antibodies to total FAK (clone 4.47; Upstate), total Src (clone L4A1; Cell Signaling), total Akt (Cell Signaling), and total paxillin (BD Biosciences - Transduction Laboratories) as well as rabbit polyclonal antibodies to phosphorylated FAK Y397 and Y576 (BD Biosciences Transduction Laboratories), phosphorylated paxillin Y31 (BD Biosciences - Transduction Laboratories), phosphorylated Src Y416 (Cell Signaling), and phosphorylated Akt S473 (Cell Signaling) - coupled with appropriate horseradish peroxidase-conjugated secondary antibodies (Cell Signaling) - were used for immunodetection of blotted proteins. Bands were detected with enhanced chemiluminescence (GE Healthcare) using a Kodak Image Station 440CF (Perkin Elmer).

MTT cell proliferation assay. Isolated tumor cells were seeded at $10^{4}$ cells/ well on collagen I-coated 96-well plates. Proliferation was assessed by colorimetric analysis of MTT (ATCC) reduction every 24 hours. Absorbance values were obtained at a wavelength of $570 \mathrm{~nm}$ by spectrophotometer.

BrdU incorporation. In vivo tumor BrdU incorporation was assessed by i.p. injection of $2 \mathrm{mg} \mathrm{BrdU} 12$ hours before tumor resection. Isolated tumor cell suspensions were fixed, permeabilized, and stained with a fluorochrome-conjugated anti-BrdU antibody according to the manufacturer's protocol (BD Biosciences - Pharmingen). BrdU incorporation was determined by flow cytometric analysis using a FACSCalibur flow cytometer (BD Biosciences) and WinMDI software (version 2.9; http:// facs.scripps.edu/software.html).

Immunohistochemistry. Full-thickness surgical wounds were excised 12 hours after exposure to HA-expressing CT26 tumor cells, fixed in 10\% formalin for 24 hours, and embedded in paraffin. Sections of $5 \mu \mathrm{m}$ through the wound were placed on slides, deparaffinized, rehydrated, and analyzed by immunohistochemistry with anti-HA antibody (clone 6E2; Cell Signaling) followed by Vectastain Universal ABC kit (Vector Labs). Histological sections of $\mathrm{Co} 26$ tumors 20 days after implantation were similarly prepared. Proliferating cells were detected using a Proliferating Cell Nuclear Antigen kit (Zymed). Sections were counterstained with hematoxylin for histological orientation and photographed on a Nikon Microphot-FXA.

Cell invasion assay. Isolated tumor cells were assessed for their ability to migrate through a collagen I-coated membrane over a 24-hour time period in a simplified Boyden chamber per the manufacturer's protocol (Trevigen). Briefly, tumor cells were seeded in growth medium containing $10 \% \mathrm{FBS}$, and bottom chambers were filled with serum-free medium. After 24 hours, invasive cells were stained with Calcein AM and detected by a fluorescent plate reader.

Statistics. Data represent mean \pm SEM and were analyzed by paired Student's $t$ test, Wilcoxon matched-pairs signed-ranks, or log-rank test as appropriate. The Sidak correction was used for multiple comparisons (58). The desired level of statistical significance was set a priori as $95 \%$ confidence.

\section{Acknowledgments}

Erin Heard, Ali Elhorr, and Ehab El Akkary provided technical assistance. This work was supported in part by NIH grant RO1DK06771 and a VA Merit Review (to M.D. Basson).

Received for publication October 19, 2007, and accepted in revised form July 9, 2008.

Address correspondence to: Marc D. Basson, Surgical Service, John D. Dingell VA Medical Center, 4646 John R. Street, Detroit, Michigan 48201-1932, USA. Phone: (313) 576-3598; Fax: (313) 576-1002; E-mail: marc.basson@va.gov. 
1. Sugarbaker, P.H. 1999. Successful management of microscopic residual disease in large bowel cancer. Cancer Chemother. Pharmacol. 43(Suppl.):S15-S25.

2. Umpleby, H.C., Fermor, B., Symes, M.O., and Williamson, R.C. 1984. Viability of exfoliated colorectal carcinoma cells. Br. J. Surg. 71:659-663.

3. Fujita, S., Kudo, N., Akasu, T., and Moriya, Y. 2001. Detection of cytokeratin 19 and 20 mRNA in peripheral and mesenteric blood from colorectal cancer patients and their prognosis. Int. J. Colorectal Dis. 16:141-146.

4. Guller, U., et al. 2002. Disseminated single tumor cells as detected by real-time quantitative polymerase chain reaction represent a prognostic factor in patients undergoing surgery for colorectal cancer. Ann. Surg. 236:768-775; discussion 775-766.

5. Hughes, E.S., McDermott, F.T., Polglase, A.L., and Johnson, W.R. 1983. Tumor recurrence in the abdominal wall scar tissue after large-bowel cancer surgery. Dis. Colon Rectum. 26:571-572.

6. Allardyce, R., Morreau, P., and Bagshaw, P. 1996 Tumor cell distribution following laparoscopic colectomy in a porcine model. Dis. Colon Rectum. 39:S47-S52.

7. Reilly, W.T., et al. 1996. Wound recurrence following conventional treatment of colorectal cancer. A rare but perhaps underestimated problem. Dis. Colon Rectum. 39:200-207.

8. Clinical Outcomes of Surgical Therapy Study Group. 2004. A comparison of laparoscopically assisted and open colectomy for colon cancer. N. Engl. J. Med. 350:2050-2059.

9. Turnbull, R.B., Jr., Kyle, K., Watson, F.R., and Spratt, J. 1967. Cancer of the colon: the influence of the no-touch isolation technic on survival rates. Ann. Surg. 166:420-427.

10. Basson, M.D., et al. 2000. Effects of increased ambient pressure on colon cancer cell adhesion. J. Cell. Biochem. 78:47-61.

11. van der Voort van Zyp, J., Conway, W.C., Thamilselvan, V., Polin, L., and Basson, M.D. 2005. Divalent cations influence colon cancer cell adhesion in a murine transplantable tumor model. Am. J. Surg. 190:701-707.

12. Thamilselvan, V., and Basson, M.D. 2004. Pressure activates colon cancer cell adhesion by inside-out focal adhesion complex and actin cytoskeletal signaling. Gastroenterology. 126:8-18

13. Thamilselvan, V., Craig, D.H., and Basson, M.D 2007. FAK association with multiple signal proteins mediates pressure-induced colon cancer cell adhesion via a Src-dependent PI3K/Akt pathway. FASEB J. 21:1730-1741.

14. Thamilselvan, V., Patel, A., van der Voort van Zyp, J., and Basson, M.D. 2004. Colon cancer cell adhesion in response to Src kinase activation and actincytoskeleton by non-laminar shear stress. J. Cell. Biochem. 92:361-371.

15. van Zyp, J.V., et al. 2006. Extracellular pressure stimulates tumor cell adhesion in vitro by paxillin activation. Cancer Biol. Ther. 5:1169-1178.

16. Less, J.R., et al. 1992. Interstitial hypertension in human breast and colorectal tumors. Cancer Res. 52:6371-6374.

17. Nathan, S.S., et al. 2005. Elevated physiologic tumor pressure promotes proliferation and chemosensitivity in human osteosarcoma. Clin. Cancer Res. 11:2389-2397.

18. Dregelid, E., and Svendsen, E. 1988. Endothelial cell injury in human saphenous veins after manipulation and tweezer grasping. J. Cardiovasc. Surg. (Torino). 29:464-469.

19. Wu, J.S., et al. 1997. Implantation of colon cancer at trocar sites is increased by low pressure pneumoperitoneum. Surgery. 122:1-7.

20. Moore-Olufemi, S.D., et al. 2005. Effects of pri- mary and secondary intra-abdominal hypertension on mesenteric lymph flow: implications for the abdominal compartment syndrome. Shock. 23:571-575.

21. Conway, W.C., et al. 2006. Paxillin modulates squamous cancer cell adhesion and is important in pressure-augmented adhesion. J. Cell. Biochem. 98: $1507-1516$

22. Downey, C., et al. 2006. Pressure stimulates breast cancer cell adhesion independently of cell cycle and apoptosis regulatory protein (CARP)-1 regulation of focal adhesion kinase. Am. J. Surg. 192:631-635.

23. Thamilselvan, V., and Basson, M.D. 2005. The role of the cytoskeleton in differentially regulating pressure-mediated effects on malignant colonocyte focal adhesion signaling and cell adhesion. Carcinogenesis. 26:1687-1697.

24. Downing, K.H. 2000. Structural basis for the interaction of tubulin with proteins and drugs that affect microtubule dynamics. Annu. Rev. Cell Dev. Biol. 16:89-111.

25. van der Voort van Zyp, J., Thamilselvan, V., Walsh, M., Polin, L., and Basson, M.D. 2004. Extracellular pressure stimulates colon cancer cell adhesion in vitro and to surgical wounds by Src (sarcoma protein) activation. Am. J. Surg. 188:467-473.

26. Usui, T. 2007. Actin- and microtubule-targeting bioprobes: their binding sites and inhibitory mechanisms. Biosci. Biotechnol. Biochem. 71:300-308.

27. Hiromura, M., et al. 2004. Inhibition of Akt kinase activity by a peptide spanning the betaA strand of the proto-oncogene TCL1. J. Biol. Chem. 279:53407-53418.

28. Li, Y.S., Haga, J.H., and Chien, S. 2005. Molecular basis of the effects of shear stress on vascular endothelial cells. J. Biomech. 38:1949-1971.

29. Shin, H.Y., Gerritsen, M.E., and Bizios, R. 2002 Regulation of endothelial cell proliferation and apoptosis by cyclic pressure. Ann. Biomed. Eng. 30:297-304.

30. Ashida, N., Takechi, H., Kita, T., and Arai, H. 2003. Vortex-mediated mechanical stress induces integrin-dependent cell adhesion mediated by inositol 1,4,5-trisphosphate-sensitive Ca2 + release in THP-1 cells. J. Biol. Chem. 278:9327-9331.

31. Haier, J., and Nicolson, G.L. 2001. Tumor cell adhesion under hydrodynamic conditions of fluid flow. APMIS. 109:241-262

32. Atance, J., Yost, M.J., and Carver, W. 2004. Influence of the extracellular matrix on the regulation of cardiac fibroblast behavior by mechanical stretch. J. Cell. Physiol. 200:377-386.

33. Yang, F., and Li, Y.H. 2002. Roles of integrins and cytoskeleton in cellular mechanotransduction [In Chinese]. Space Med. Med. Eng. (Beijing). 15:309-312.

34. Huynh, T.T., et al. 1999. External support modulates $\mathrm{G}$ protein expression and receptor coupling in experimental vein grafts. Surgery. 126:127-134.

35. Ghazi, A., Berrier, C., Ajouz, B., and Besnard, M. 1998. Mechanosensitive ion channels and their mode of activation. Biochimie. 80:357-362.

36. Katsumi, A., Orr, A.W., Tzima, E., and Schwartz, M.A. 2004. Integrins in mechanotransduction. J. Biol. Chem. 279:12001-12004.

37. Ingber, D.E. 1997. Tensegrity: the architectural basis of cellular mechanotransduction. Annu. Rev. Physiol. 59:575-599.

38. Ingber, D.E. 2003. Tensegrity II. How structural networks influence cellular information processing networks. J. Cell. Sci. 116:1397-1408.

39. Walsh, M.F., Woo, R.K., Gomez, R, and Basson, M.D. 2004. Extracellular pressure stimulates colon cancer cell proliferation via a mechanism requiring $\mathrm{PKC}$ and tyrosine kinase signals. Cell Prolif. 37:427-441.

40. Wang, S., and Basson, M.D. 2008. Identification of functional domains in $\mathrm{AKT}$ responsible for distinct roles of AKT isoforms in pressure-stimulated cancer cell adhesion. Exp. Cell Res. 314:286-296.

41. Hu, Y.L., et al. 2006. Roles of microfilaments and microtubules in paxillin dynamics. Biochem. Biophys. Res. Commun. 348:1463-1471.

42. Craig, D.H., Haimovich, B., and Basson, M.D. 2007. Alpha-actinin-1 phosphorylation modulates pressure-induced colon cancer cell adhesion through regulation of focal adhesion kinase-Src interaction. Am. J. Physiol. Cell Physiol. 293:C1862-C1874.

43. Perkins, G.L., Derfoul, A., Ast, A., and Hall, D.J. 2005. An inhibitor of the stretch-activated cation receptor exerts a potent effect on chondrocyte phenotype. Differentiation. 73:199-211.

44. Alenghat, F.J., Nauli, S.M., Kolb, R., Zhou, J., and Ingber, D.E. 2004. Global cytoskeletal control of mechanotransduction in kidney epithelial cells. Exp. Cell Res. 301:23-30.

45. Mohri, Y., Tonouchi, H., Kobayashi, M., Nakai, K., and Kusunoki, M. 2007. Randomized clinical trial of single- versus multiple-dose antimicrobial prophylaxis in gastric cancer surgery. Br. J. Surg. 94:683-688.

46. Feng, G., and Kaplowitz, N. 2000. Colchicine protects mice from the lethal effect of an agonistic anti-Fas antibody. J. Clin. Invest. 105:329-339.

47. Reddy, R.N., Latendresse, J.R., and Mehendale, H.M. 2006. Colchicine antimitosis causes progression of S-(1,2-dichlorovinyl)-L-cysteine-induced injury leading to acute renal failure and death in mice. Toxicology. 220:147-159.

48. Aguirre-Cruz, L., and Sotelo, J. 1998. Lack of therapeutic effect of colchicine on murine toxoplasmosis. J. Parasitol. 84:163-164.

49. Banan, A., McCormack, S.A., and Johnson, L.R. 1998. Polyamines are required for microtubule formation during gastric mucosal healing. Am. J. Physiol. 274:G879-G885.

50. Dudkiewicz, I., Brosh, T., Perelman, M., and Salai, M. 2005. Colchicine inhibits fracture union and reduces bone strength--in vivo study. J. Orthop. Res. 23:877-881.

51. Lee, J.S., and Gotlieb, A.I. 2005. Microtubules regulate aortic endothelial cell actin microfilament reorganization in intact and repairing monolayers. Histol. Histopathol. 20:455-465.

52. Gordon, S.R., and Buxar, R.M. 1997. Inhibition of cytoskeletal reorganization stimulates actin and tubulin syntheses during injury-induced cell migration in the corneal endothelium. J. Cell. Biochem. 67:409-421.

53. Sayfan, J., Averbuch, F., Koltun, L., and Benyamin, N. 2000. Effect of rectal stump washout on the presence of free malignant cells in the rectum during anterior resection for rectal cancer. Dis. Colon Rectum. 43:1710-1712.

54. da Silva, R.G., and Sugarbaker, P.H. 2006. Analysis of prognostic factors in seventy patients having a complete cytoreduction plus perioperative intraperitoneal chemotherapy for carcinomatosis from colorectal cancer. J. Am. Coll. Surg. 203:878-886.

55. Katz, M.H., and Barone, R.M. 2003. The rationale of perioperative intraperitoneal chemotherapy in the treatment of peritoneal surface malignancies. Surg. Oncol. Clin. N. Am. 12:673-688.

56. Griswold, D.P., and Corbett, T.H. 1975. A colon tumor model for anticancer agent evaluation. Cancer. 36:2441-2444.

57. Hazeldine, S.T., et al. 2001. Design, synthesis, and biological evaluation of analogues of the antitumor agent, 2-(4-[(7-chloro-2-quinoxalinyl)oxy] phenoxy)propionic acid (XK469). J. Med. Chem. 44:1758-1776

58. Sankoh, A.J., Huque, M.F., and Dubey, S.D. 1997. Some comments on frequently used multiple endpoint adjustment methods in clinical trials. Stat. Med. 16:2529-2542. 